\title{
CARACTERIZAÇÃO BIOESTRATIGRÁFICA DO NEÓGENO SUPERIOR DA BACIA DE SANTOS COM BASE EM FORAMINÍFEROS PLANCTÔNICOS
}

\author{
RODRIGO DA COSTA PORTILHO-RAMOS \\ Programa de Pós-Graduação em Geoquímica Ambiental, Departamento de Geoquímica, UFF, Outeiro de São João Batista s/n, \\ Centro, 24020-007, Niterói, RJ, Brasil.rcpramos@yahoo.com.br \\ ARISTÓTELES DE MORAES RIOS-NETTO \\ Laboratório de Bioestratigrafia, Paleoambientes e Paleoclima, Departamento de Geologia, IGEO, UFRJ, Cidade Universitária, \\ Ilha do Fundão, 21949-900, Rio de Janeiro, RJ, Brasil.rios.netto@geologia.ufrj.br

\section{CÁTIA FERNANDES BARBOSA} \\ Programa de Pós-Graduação em Geoquímica Ambiental, Departamento de Geoquímica, UFF, Outeiro de São João Batista s/n, \\ Centro, 24020-007, Niterói, RJ, Brasil.catia@geoq.uff.br
}

RESUMO - A margem continental brasileira tem apresentado importante reservas de hidrocarbonetos do país e, por isso, vem sendo alvo de diversas pesquisas relacionadas à bioestratigrafia, à paleoecologia e à paleoceanografia. A caracterização bioestratigráfica de rochas do Neógeno superior (Pleistoceno/Holoceno) possibilita o reconhecimento de um arcabouço cronoestratigráfico para uma área e contribui para a identificação de lacunas e hiatos, além de possíveis escorregamentos e retrabalhamentos. Assim, esse procedimento tem se mostrado de grande utilidade no estudo da estabilidade de áreas do talude continental, contribuindo na implantação de plataformas de perfuração e dutos submarinos de óleo/gás. No presente trabalho, foram estudados os $10 \mathrm{~m}$ superiores do testemunho JPC-95, perfurado na porção sul da bacia de Santos, Brasil. Foram reconhecidas as biozonas X (Pleistoceno), Y (Pleistoceno) e Z (Holoceno), estando seus limites posicionados entre as amostras 920-921 cm e 910-911 cm, e $20-21 \mathrm{~cm}$ e $10-11$ $\mathrm{cm}$, respectivamente. Foram identificadas também as subzonas X1 e Y5 a Y1. Quatro bioeventos foram reconhecidos, com idade absoluta bem conhecida na literatura especializada: (i) o primeiro desaparecimento do plexo Pulleniatina obliquiloculata (YP.1; $84 \mathrm{Ka}$ ); (ii) o segundo desaparecimento do plexo Pulleniatina obliquiloculata (YP.2; 67,7 Ka$74,4 \mathrm{Ka}$ ); (iii) o último desaparecimento do plexo Pulleniatina obliquiloculata (YP.3, 42-45 Ka); e (iv) o último reaparecimento do plexo menardiforme $(11,5 \mathrm{Ka})$. Estes resultados permitiram o refinamento bioestratigráfico para o intervalo do Neógeno Superior da bacia de Santos com base em foraminíferos e a correlação cronoestratigráfica com bioeventos reconhecidos na literatura para a bacia de Campos, possibilitando o reconhecimento de uma sequiência estratigráfica sem hiatos na sedimentação permitindo verificar a ausência de turbiditos ou escorregamentos no testemunho.

Palavras-chave: Neógeno superior, bioestratigrafia, foraminíferos planctônicos, bacia de Santos, Brasil, Atlântico Sul.

ABSTRACT - BIOESTRATIGRAPHIC CHARACTERIZATION OF UPPER NEOGENE OF SANTOS BASIN BASED ON PLANKTONIC FORAMINIFERA. The Brazilian continental margin has presented important hydrocarbon reservoirs of the country, and therefore it has been a target of several researches related to bioestratigraphy, paleecology and paleoceanography. The bioestratigraphic characterization of the upper Neogene (Pleistocene/Holocene) enables chronostratigraphic framework of the studied area, where, besides the identification of gaps and hiatuses, it is a fundamental tool in continental slope stability studies, playing an important role for platforms and submarine pipelines locations. The present work studied the uppermost $10 \mathrm{~m}$ of the piston core JPC-95, drilled in the southeastern area of the Santos Basin, Brazil. We recognized the biozones X, Y and Z, which limits are positioned between the sampling intervals 920-921 cm and 910-911 cm, and 20-21 and 10-11 cm, respectively. We have also identified the subzones X1 and Y5 to Y1. Four bioevents were recognized, with well known absolute age dating in the literature: (i) the first regional disappearance level of Pulleniatina obliquiloculata plexus (biohorizon YP.1; $84 \mathrm{Ka}$ ); (ii) the second regional disappearance level of Pulleniatina obliquiloculata plexus (biohorizon YP.2; 67,7 - 74,4 Ka); (iii) the last regional disappearance level of Pulleniatina obliquiloculata plexus (biohorizon YP.3; $84 \mathrm{Ka}$.); and (iv) the last reappearance of the menardiform plexus $(11,5 \mathrm{Ka})$. These results allow the bioestratigraphical refining to the Neogene interval of Santos basin based on foraminifera and the cronoestratigraphical correlation with bioevents that are recognized in the literature to Campos Basin, turning possible to recognize a stratigraphical sequence without gaps on the sedimentation, which allows verify the absence of turbidites or creeps on the core.

Key words: Upper Neogene, bioestratigraphy, planktonic foraminifera, Santos Basin, Brazil, southwestern Atlantic Ocean. 


\section{INTRODUÇÃO}

A caracterização bioestratigráfica de rochas do Neógeno superior (Pleistoceno/Holoceno) é ferramenta fundamental no estudo da estabilidade do talude continental, contribuindo na implantação segura de plataformas de perfuração e dutos submarinos de óleo/gás. Ela possibilita o reconhecimento de um arcabouço biocronoestratigráfico para uma área e contribui para a identificação de lacunas e hiatos, além de possíveis escorregamentos e retrabalhamentos, que poderiam afetar a segurança dessas instalações. Devido a sua importância no cenário nacional, em termos de hidrocarbonetos, a margem continental brasileira vem sendo alvo de diversas pesquisas relacionadas à bioestratigrafia, à paleoecologia e à paleoceanografia, principalmente de intervalos pré-pliocênicos, sendo ainda poucos os estudos dessa natureza realizados em sedimentos do Neógeno superior. O presente trabalho pretende contribuir com essa demanda a partir de estudos bioestratigráficos baseados em foraminíferos planctônicos. Além da aplicação em geotecnia, esse estudo contribui também para o entendimento da evolução do paleoclima e da paleoceanografia do Atlântico Sul durante o Pleistoceno/Holoceno. O objetivo específico é desenvolver uma moldura bioestratigráfica para os $10 \mathrm{~m}$ superiores do testemunho JPC-95, coletado na bacia de Santos, que permita a observação do seqüenciamento dos eventos ocorridos na área e sua futura correlação com dados advindos de estudos de outros testemunhos coletados na região.

\section{ÁREA DE ESTUDO}

A bacia de Santos ocupa uma área de $275.000 \mathrm{~km}^{2}$ e está localizada na margem continental sudeste-sul brasileira, entre os paralelos $23^{\circ}$ e $28^{\circ}$ Sul. Seu limite norte com a bacia de Campos é marcado pelo alto de Cabo Frio, enquanto o Alto de Florianópolis marca o limite sul com a bacia de Pelotas (Pereira \& Feijó, 1994). O limite oeste é dado pelas serras do Mar e da Mantiqueira e pelo maciço da Carioca (Macedo, 1989; Mesquita, 1995). A leste, a bacia de Santos está em continuidade estrutural e estratigráfica com o platô de São Paulo (Macedo, 1989). O limite batimétrico da bacia de Santos é considerado atualmente em cerca de $2.000 \mathrm{~m}$ de lâmina d'água.

\section{MATERIAL E MÉTODOS}

As amostras estudadas no presente trabalho foram coletadas em 1998, durante o cruzeiro KNORR 159-5 do Woods Hole Oceanographic Institution (WHOI; Massachusetts, EUA), que realizou diversas perfurações no talude dessa região, incluindo o platô de São Paulo e a bacia de Santos, com o objetivo de ampliar os conhecimentos sobre a evolução paleoceanográfica do Atlântico Sul. Coletado na porção sul da bacia de Santos (27052,73'S, 46 55 ,25'W, Figura 1), sob lâmina d'água de $1.485 \mathrm{~m}$, o testemunho a pistão JPC 95 alcançou $18 \mathrm{~m}$ de comprimento, dos quais os $10 \mathrm{~m}$ superiores foram analisados no presente estudo, num total de 100 amostras.
Em laboratório, as amostras foram pesadas (1 g cada) e lavadas sob água corrente, em peneira com malha $62 \mu \mathrm{m}$ e, posteriormente, secadas em estufa com temperatura máxima de $60^{\circ} \mathrm{C}$. Em seguida, cada amostra foi peneirada a seco em peneiras com malha de 125 e $250 \mu \mathrm{m}$, para triagem e contagem de cerca 300 espécimes de foraminíferos planctônicos. Os cálculos de porcentagem foram feitos para cada táxon significativo de foraminíferos, em termos paleoceanográficos e/ou paleoclimáticos, de acordo com a bibliografia especializada. As carapaças foram analisadas sob estereomicroscópio, com aumentos variando de 6 a $66 \mathrm{X}$. A identificação dos táxons tomou como base os trabalhos de Bolli \& Saunders (1985) e Stainforth et al. (1975), entre outros. A bioestratigrafia (de alta resolução para o Pleistoceno/Holoceno) baseou-se principalmente nos trabalhos de Ericson \& Wollin (1968) e Vicalvi (1997, 1999), levando em conta dados regionais de aparecimentos, desaparecimentos e reaparecimentos, assim como a abundância relativa de táxons de foraminíferos planctônicos altamente sensíveis a oscilações climáticas. O biozoneamento de Ericson \& Wollin (1968) correlaciona as variações climáticas com a freqüência de Globorotalia menardii s.l., espécie tolerante a águas quentes, dividindo o Pleistoceno/Holoceno em dez biozonas (R a Z), de acordo com a presença/ausência desse táxon (presença $=$ intervalos interglaciais; ausência = intervalos glaciais). As biozonas X, Y eZ foram subdivididas em 18 subzonas por Vicalvi $(1997,1999)$.

O termo "plexo" se refere a um grupo de espécies e/ou subespécies de morfologia semelhante e que apresentam a mesma reação as variações ambientais (sensu Vicalvi, 1997, 1999). A expressão "plexo menardiforme" inclui os táxons Globorotalia menardii menardii, G. menardii cultrata, G. fimbriata, G. flexuosa e G. tumida tumida, formando o principal grupo indicador de águas quentes. O "plexo Pulleniatina" compreende o conjunto das espécies Pulleniatina primalis, $P$. obliquiloculata e $P$. finalis, sendo considerado por muitos autores, como representante de águas quentes, porém com maior tolerância a baixas temperaturas do que o plexo menardiforme. Por ter um comportamento mais euritérmico, tolerando águas mais frias do que os representantes do plexo menardiforme, tende a desaparecer mais tardiamente, nos momentos de transição de períodos interglaciais para glaciais, e reaparecer antes, quando um novo intervalo interglacial se aproxima (Prell \& Damuth, 1978).

\section{RESULTADOS E DISCUSSÃO}

As carapaças de foraminíferos planctônicos encontradas nas amostras estudadas apresentam excelente estado de preservação, o que facilitou sua contagem e identificação taxonômica. Por se tratar de uma área sob influência da corrente do Brasil, a maior parte dos táxons encontrados no testemunho é característica de zonas subtropicais, entre eles Globigerinoides ruber, G. trilobus s.l., G. conglobatus, Neogloboquadrina dutertrei s.l., Hastigerina siphonifera, H. aequilateralis, Orbulina universa, Globigerina rubescens o plexo menardiforme e o plexo Pulleniatina (Boltovskoy et al., 1996). No entanto, representantes da corrente das 
Malvinas também foram encontrados, entre eles Globigerina bulloides, Globorotalia scitula scitula, G. truncatulinoides s.l., além de G. inflata, espécie considerada de águas frias, mas que, segundo Boltovskoy et al. (1996), habita regiões transicionais de médias latitudes. Além das espécies citadas acima, também foram encontradas Globigerinoides saculiferus, Globigerina calida calida, Tinophodella ambitacrena, Globigerinita naparimaesnsis, Globorotalia crassaformis s.l., Turborotalita humilis e Candeina nitida.

Como pode ser observado na Figura 2, o intervalo que se estende da amostra 990-991 cm até a amostra 920-921 cm, totalizando $70 \mathrm{~cm}$ de espessura, foi reconhecido como pertencente à biozona X, de Ericson \& Wollin (1968), conforme evidenciado pela presença do plexo menardiforme, incluindo Globorotalia flexuosa, espécie característica dessa biozona. A presença desse plexo revela que os sedimentos desse intervalo foram depositados sob condições de águas quentes (último intervalo interglacial), e sua alta porcentagem (entre 0.9 e $12.1 \%$ ) em relação à biozona $\mathrm{Z}$ (que será descrita mais adiante), aponta para um intervalo onde o clima foi mais quente do que o atual (Vicalvi, 1999). Nessa biozona, foi reconhecida somente a subzona X1, de Vicalvi (1997); a confirmação ou não da presença das demais subzonas da biozona $\mathrm{X}$ depende do estudo do restante do testemunho.

A ausência do plexo menardiforme entre as profundidades 911 e $20 \mathrm{~cm}$ do testemunho, com uma rara ocorrência na amostra 90-91 cm, indica a presença da biozona Y de Ericson \& Wollin (1968) e revela a deposição em um ambiente de clima frio, caracterizando o último episódio glacial, ocorrido no Pleistoceno final. Essa biozona é a mais longa do intervalo estudado, com 8,9 m de espessura. Em sua base pode-se reconhecer um nível de desaparecimento local do plexo Pulleniatina. Ao longo da biozona Y, esse táxon reaparece e desaparece localmente mais duas vezes, permitindo reconhecer as cinco subzonas propostas por Vicalvi (1999) para a biozona Y (Y1, Y2, Y3, Y4 e Y5). Cabe lembrar que Prell \& Damuth (1978) haviam reconhecido um único nível de desaparecimento do plexo Pulleniatina, ao qual denominaram "biohorizonte Pulleniatina obliquiloculata"; esse biohorizonte tem extensão global, com razoável sincronismo regional (Bé et al., 1976; Prell \& Damuth, 1978), e foi observado também por Vicalvi (1999) e Sanjinés (2004) na bacia de

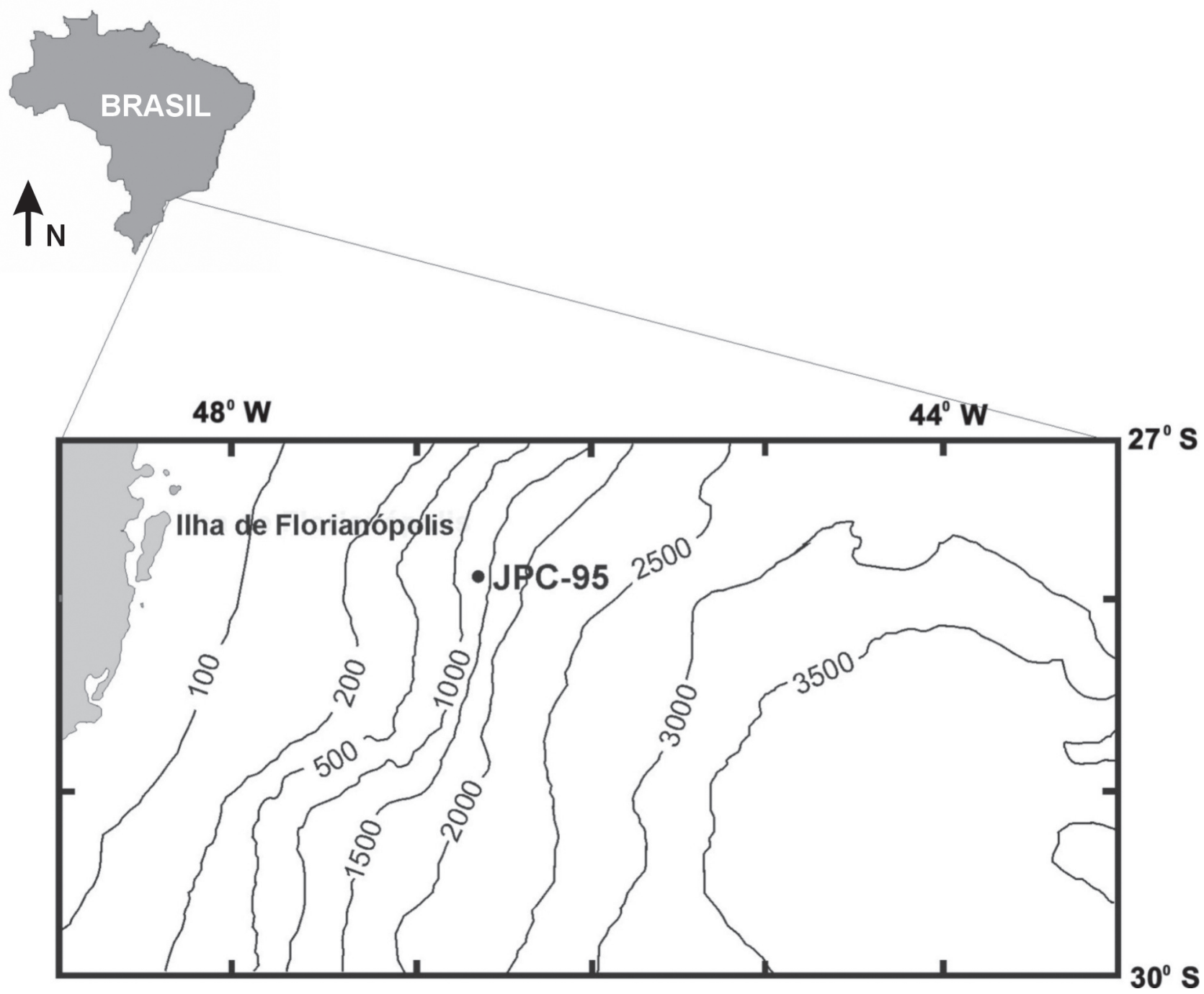

Figura 1. Mapa de localização do testemunho JPC-95 (modificado de Kumar et al., 1977).

Figure 1. Location map of the core JPC-95 (modified from Kumar et al., 1977). 
Campos e por Portilho-Ramos \& Rios-Netto (2002) e PortilhoRamos (2003) na bacia de Santos. No entanto, além do biohorizonte Pulleniatina obliquiloculata de Prell \& Damuth (1978), Vicalvi (1999) reconheceu outros dois níveis de desaparecimento do gênero Pulleniatina, na base e na parte inferior da biozona $\mathrm{Y}$, o que lhe permitiu dividir essa biozona em cinco subzonas. O primeiro nível de desaparecimento desse plexo foi denominado YP.1 (primeiro biohorizonte Pulleniatina obliquiloculata) e corresponde ao limite entre as zonas X e Y de Ericson \& Wollin (1968), datado em 84.000 anos. O segundo biohorizonte Pulleniatina obliquiloculata, denominado YP.2, marca o limite entre as subzonas Y4/Y3 de Vicalvi (1999), com idade absoluta variando de 67.700 e 74.400 anos. O terceiro biohorizonte Pulleniatina obliquiloculata (YP.3), tem idades absolutas variando entre 42.000 e 45.000 anos e marca o limite entre as subzonas $\mathrm{Y} 2 / \mathrm{Y} 1$ do mesmo autor. Segundo Bé et al. (1976), o gênero Pulleniatina reaparece em sedimentos do Atlântico equatorial e do Caribe na porção final da biozona Y (Pleistoceno), há aproximadamente 15.000 anos, tornando-se abundante durante o Holoceno. No entanto, assim como Vicalvi (1999), o presente trabalho observa um retorno moderado do plexo
Pulleniatina somente na base da subzona Z2 (no limite Holoceno/Pleistoceno), há cerca de 11.000 anos.

A partir dessas observações, o intervalo bioestratigráfico entre as amostras 910-911 a 800-801 cm foi reconhecido como subzona Y5 de Vicalvi (1999), caracterizada pela ausência do plexo Pulleniatina. Seu limite inferior, com a subzona X1, é marcado pelo biohorizonte YP.1, encontrado entre as profundidades 921 e $910 \mathrm{~cm}$, com idade absoluta de $84 \mathrm{Ka}$. Seu limite superior é dado pelo reaparecimento do plexo Pulleniatina (Figura 2).

O retorno do plexo Pulleniatina no intervalo entre as amostras 790-791 e 670-671 cm caracteriza a subzona Y4, estando seu limite inferior, com a subzona Y5, entre as amostras 800-801 e 790-791 cm, marcado pelo reaparecimento desse táxon. $\mathrm{O}$ topo dessa subzona é dado pelo biohorizonte YP.2, com idade entre 67.7 e $74.4 \mathrm{Ka}$. A presença do plexo Pulleniatina revela, segundo Vicalvi (1999), um intervalo de tempo em que o clima da região foi menos frio, capaz de abrigar esse táxon que é menos sensível à temperatura do que o plexo menardiforme.

O intervalo bioestratigráfico que se estende da amostra 660-661 até $260-261 \mathrm{~cm}$ foi reconhecido como pertencente à

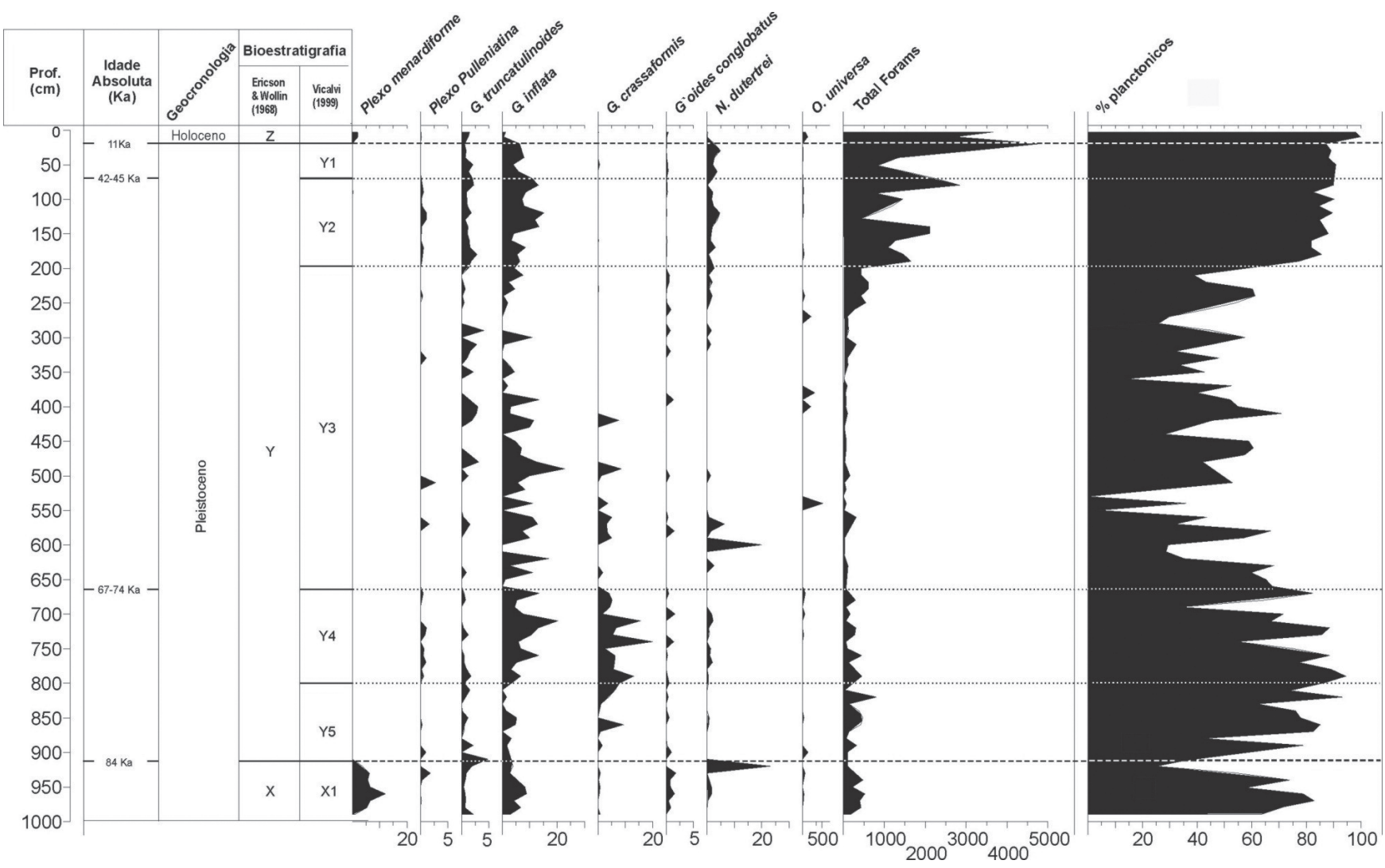

Figura 2. Carta estratigráfica do intervalo estudado do testemunho JPC-95, mostrando a freqüência dos táxons analisados, o número total de carapaças de foraminíferos planctônicos/g e o percentual de foraminíferos planctônicos/amostra. Biozonas reconhecidas: Z (Holoceno), Y (último glacial do Pleistoceno) e X (último interglacial do Pleistoceno) (Ericson \& Wollin, 1968), pela presença/ausência do plexo menardiforme; subzonas Y1 a Y5 (Vicalvi, 1999), pela ocorrência dos três níveis de desaparecimento do plexo Pulleniatina (biohorizontes YP1, YP2 e YP3).

Figure 2. Stratigraphic chart of the studied interval of the core JPC-95, showing the percentage of the analyzed taxa, the total number of foraminiferal tests/g, and the percentage of planktonic foraminifera/sample. Recognized biozones: $Z$ (Holocene), $Y$ (Pleistocene last glacial) and X (Pleistocene last interglacial) (Ericson \& Wollin, 1968), by the presence/absence of menadiformi plexus; subzones Y1 a Y5 (Vicalvi, 1999), by the occurrence of three levels of Pulleniatina plexus disappearance (biohorizontes YP1, YP2 e YP3). 
subzona Y3, evidenciado pela ausência do plexo Pulleniatina. Seu limite inferior, com a subzona Y4, é dado pelo biohorizonte YP.2, enquanto seu limite superior, com a subzona Y2, encontra-se entre as amostras 200-201 e 190-191 cm, marcado por novo reaparecimento do plexo Pulleniatina.

A subzona Y2 foi reconhecida entre as amostras 190-191 e 70-71 cm. Sua base é identificada pelo nível de retorno do plexo Pulleniatina, entre as amostras 200-201 e 190-191 cm, enquanto seu limite superior, entre as profundidades $71 \mathrm{e}$ $60 \mathrm{~cm}$, é definido pelo biohorizonte YP3, com idade absoluta estimada em 42-45 Ka. Essa subzona, observada também por Vicalvi (1999), é marcada pelo retorno expressivo do plexo Pulleniatina, que atinge os valores mais altos de toda zona Y (entre 0.2 e $2.2 \%$ ), sugerindo um pequeno aquecimento das águas superficiais dessa região, durante a última glaciação.

O intervalo que se estende da amostra 60-61 cm até a amostra $20-21 \mathrm{~cm}$, totalizando $40 \mathrm{~cm}$ de espessura, foi reconhecido como pertencente à subzona $\mathrm{Y} 1$, caracterizada por nova ausência do plexo Pulleniatina. A base dessa subzona, entre as amostras 70-71 e 60-61 cm, é indicada pelo biohorizonte YP3, e marca o último desaparecimento desse plexo ocorrido há 42-45 Ka. Seu limite superior, com a biozona Z, de Ericson \& Wollin (1968), encontra-se entre os intervalos 20-21 cm e 10-11 cm, e é caracterizado pelo reaparecimento do plexo menardiforme, na ausência de Globorotalia flexuosa (Figura 2). Essa subzona representa o final da última glaciação ocorrida durante o Pleistoceno.

A biozona Z (Holoceno) foi encontrada a partir de $11 \mathrm{~cm}$ do topo do testemunho, sendo seu limite inferior indicado pelo retorno do plexo menardiforme (com ausência de Globorotalia flexuosa), com porcentagens entre 1.9 e $2.1 \%$, além da presença do plexo Pulleniatina. Seu limite inferior, com a biozona Y, encontra-se entre as amostras 20-21 e 10-11 $\mathrm{cm}$ e marca o limite Pleistoceno/Holoceno, datado em $11 \mathrm{Ka}$. A presença dos plexos menardiforme e de Pulleniatina nesse intervalo indicam o retorno das águas oceânicas quentes depois do último episódio glacial. Não foi possível o reconhecimento das subzonas Z2 e Z1, de Vicalvi (1997), o que pode ser devido à baixa taxa de sedimentação desse intervalo de tempo ou à insuficiência no espaçamento da amostragem realizada, uma vez que somente duas amostras $(10-11 \mathrm{~cm}$ e $3-4 \mathrm{~cm})$ foram coletadas nesse intervalo.

\section{CONCLUSÕES}

Foram reconhecidas no intervalo estudado do testemunho JPC 95, as biozonas Z (Holoceno), Y (último glacial do Pleistoceno) e X (último interglacial do Pleistoceno) de Ericson \& Wollin (1968), identificadas a partir da presença/ausência do plexo menardiforme.

Foram reconhecidas as subzonas Y1 a Y5, de Vicalvi (1999), considerando-se os sucessivos desaparecimentos e reaparecimentos do plexo Pulleniatina, conforme evidenciado pelos três níveis de desaparecimento do plexo Pulleniatina (biohorizontes YP1, YP2 e YP3) propostos por aquele autor.
Somente a subzona X1 (interglacial) de Vicalvi (1997) está presente no intervalo estudado do testemunho. O reconhecimento da presença ou não das demais subzonas da biozona $\mathrm{X}$ no testemunho JPC-95 depende da continuação dos estudos no restante do testemunho.

Os resultados encontrados permitiram o refinamento bioestratigráfico para o intervalo do Neógeno Superior da bacia de Santos e a correlação cronoestratigráfica com bioeventos reconhecidos na literatura para a bacia de Campos, possibilitando o reconhecimento de uma seqüência estratigráfica sem hiatos na sedimentação permitindo verificar a ausência de turbiditos ou escorregamentos no testemunho.

Sugere-se um refinamento do espaçamento de amostragem do intervalo entre $21 \mathrm{~cm}$ e o topo do testemunho, com a finalidade de buscar reconhecer, nele, as subzonas Z1 e Z2 de Vicalvi (1999), e, na região, o nível de último reaparecimento do gênero Pulleniatina, que dataria de $15 \mathrm{Ka}$, conforme observado por Bé et al. (1976).

\section{AGRADECIMENTOS}

Agradecemos a Delia Oppo, William B. Cury, Jerry F. McManus e Ellen Roosen do Woods Hole Oceanographic Institution (EUA), pela coleta e fornecimento dos sedimentos do testemunho JPC-95. Este trabalho foi uma contribuição ao XIX Congresso Brasileiro de Paleontologia/VI Congresso Latino-americano de Paleontologia, ocorrido em Aracajú, SE, em agosto de 2005.

\section{REFERÊNCIAS}

Bé, A.W.; Damuth, J.E.; Lott. L. \& Free, R. 1976. Late Quaternary climatic record in Western Equatorial Atlantic sediment. Geological Society of America Memoir, 145:165-200.

Bolli, H.M. \& Saunders, J.B. 1985. Oligocene to Holocene low latitude planktic foraminifera. In: H.M. Bolli,; J.B. Saunders \& K. Perch-Nielsen (eds.) Plankton Stratigraphy, Cambridge University Press, p.155-262.

Boltovskoy, E.; Boltovskoy, D.; Correa, N. \& Brandini, F. 1996. Planktic foraminifera from the southwestern Atlantic $\left(30^{\circ}-60^{\circ} \mathrm{S}\right)$ : species-specific patterns in the upper $50 \mathrm{~m}$. Marine Micropaleontology, 28:53-72.

Ericson, D.B. \& Wollin, G. 1968. Pleistocene climates and chronology in deep-sea sediments. Science, 162:1227-1234.

Kumar, N.; Gamboa, L.A.P.; Schreiber, B.C. \& Mascle, J. 1977. Geologic history and origin of São Paulo Plateau (Southeastern Brazilian Margin), comparison with the Angola Margin, and the early evolution of the Northern South Atlantic. In: P.R. Supko \& K. Perch-Nielsen (eds.) Initial reports of the Deep Sea Drilling Project. Washignton, U.S. Government Printing Office, v. 39, p. 927-945.

Macedo, J.M. 1989. Evolução tectônica da bacia de Santos e áreas continentais adjacentes. Boletim de Geociências da Petrobrás. 3(3): 159-173.

Mesquita, A.C.F. 1995. Microestratigrafia do OligocenoPleistoceno na porção centro-norte da bacia de Santos, com base em foraminíferos planctônicos. Curso de Pós-graduação em Geociências, Universidade Federal do Rio Grande do Sul, Dissertação de Mestrado, 120 p. 
Pereira, M.J. \& Feijó, F.J. 1994. Bacia de Santos. Boletim de Geociências da Petrobrás, 8(1):219-234.

Portilho-Ramos, R.C. 2003. Interpretações paleoceanográficas e bioestratigráficas do testemunho JPC 17 (porção sul da bacia de Santos), com base em foraminíferos planctônicos. Escola de Ciências Biológicas e da Saúde/UNI-RIO, Monografia de Graduação, 63 p.

Portilho-Ramos, R.C. \& Rios-Netto, A.M. 2002. Bioestratigrafia do Quaternário superior no talude Sul da bacia de Santos (testemunho KNORR JPC 17), com base em foraminíferos planctônicos - resultados preliminares. Paleontologia em Destaque, 40:13.

Prell, W.L. \& Damuth, J.E. 1978. The climate-related diachrnous disappearance of Pulleniatina obliquiloculata in Late Quaternary sediments of the Atlantic and Caribbean. Marine Micropaleontology, 3(3):267-277.

Sanjinés, A.E.S. 2004. Análise bioestratigráfica de dois testemunhos a pistão do talude da bacia de Campos, RJ. Programa de
Pós-graduação em Geologia, Universidade Federal do Rio de Janeiro, Monografia de Graduação, 57 p.

Stainforth, R.M; Lamb, J.L.; Luterbacher, H.; Beard, J.H. \& Jeffords, R.M. 1975. Cenozoic planktonic foraminifera zonation and characteristics of index forms. The University of Kansas Paleontological Contributions, 2:425 (art. 62).

Vicalvi, M.A. 1997. Zoneamento bioestratigráfico e paleoclimático dos sedimentos do Quaternário Superior do talude da bacia de Campos, RJ, Brasil. Boletim de Geociências da Petrobrás, 11(1/ 2):132-165.

Vicalvi. M.A. 1999. Zoneamento bioestratigráfico e paleoclimático do Quartenário superior do talude da bacia de Campos e platô de São Paulo adjacente, com base em foraminíferos planctônicos. Programa de Pós-graduação em Geologia, Universidade Federal do Rio de Janeiro, Tese de Doutorado, 183 p.

Received in October, 2005; accepted in October, 2006 